\title{
Lifetime of electret microphones by thermal degradation analysis via electroacoustic measurements
}

\author{
E. Nogueira , Juan Sancho Gil , José Luis Sánchez Bote
}

\begin{abstract}
A B S T R A C T
Electronic equipment frequently utilises low cost electret microphones. In this paper, a complete accelerated lifetest on commercial electret microphones is presented using thermal chamber testing. The test was conducted on the microphones under study at three different temperatures. After each thermal probe, the electroacoustic characteristics of each transducer was analysed for obtaining the degradation model. In the literature such tests have been performed through charge losses on the electret materials. Life models were obtained for the degradation failures revealed from the tests and a complete temperature model was then developed, which can calculate the useful life of the microphones under any of these conditions.
\end{abstract}

\section{Introduction}

At present, more numbers of electronic equipment employ low cost electret microphones, which is not an indicator of poor quality. Nevertheless, electret microphones are available in consumer electronic gear such as telephone terminals, portable sound recorders, toys, etc. Moreover, they are extensively used as part of professional electronic equipment, as in controllers-limiters of sound pressure level, like those utilised in hospitals, pubs and small discotheques. The substantial use of these electret microphones [1] motivated us to investigate and acquire data on their quality and reliability.

In current consumer equipment, electret and MEMS microphones coexist, both operating with the electrostatic principle, which has a non-linear behaviour and needs bias to operate in a linear zone. Electret microphones use as bias permanent polarisation induced at the time of manufacture in a plastic polymer sheet, which is the electret material. This polarisation attracts the free charge on the metallic electrodes of the device, which is necessary for the proper working of principle of electrostatic transduction. As is widely acknowledged, the sensitivity of such types of microphones is proportional to the free charge induced and, therefore, to the permanent polarisation of the electret material. Therefore, any polarisation loss will, over time, result in loss of both the response and transducer performance. Analyses of the degradation of the electret materials are available in the literature [2-3]; however, identifying studies employing electroacoustic measurements is difficult. Moreover, the authors believe it could be useful to repeat the reliability analyses of consumer electret microphones to investigate how new materials can improve their behaviour despite their low price.

In this paper, three tests were performed on 30 samples of the same production batch of a single manufacturer. From among the total population, 3 subsets of 10 microphones were selected, one for each temperature. Periodically, the microphones were removed from the thermal chamber for electroacoustic measurements.

Chapter two discusses the samples and methods, and chapter three records the test results related to the variations in the frequency response of the microphones to temperature and the degradation analysis. In chapter four, the reliability and life data analysis are reported, while chapter five draws the conclusions.

\section{Samples and methods}

\subsection{Samples}

The electret microphones tested are low in cost, have high vibration 
Table 1

Manufacturer specifications of the electret microphones under test.

\begin{tabular}{ll}
\hline Specifications & Value \\
\hline Sensitivity & $-44 \pm 5 \mathrm{~dB}(0 \mathrm{~dB}=1 \mathrm{~V} / \mathrm{Pa}, 1 \mathrm{kHz})$ \\
& eq. $6.3 \mathrm{mV} / \mathrm{Pa}$. \\
Impedance & Less than $2.2 \mathrm{k} \Omega$ \\
Directivity & Omnidirectional \\
Frequency range & $20 \mathrm{~Hz}-20 \mathrm{kHz}$ \\
Max supply voltage & $10 \mathrm{~V}$ \\
Standard operating voltage & $2 \mathrm{~V}$ \\
Current consumption & Max. $0.5 \mathrm{~mA}$ \\
Sensitivity reduction & Within-3 dB and $1.5 \mathrm{~V}$ \\
S/N ratio & Less than $58 \mathrm{~dB}$ \\
Capsule diameter & $6 \mathrm{~mm}$ \\
\hline
\end{tabular}

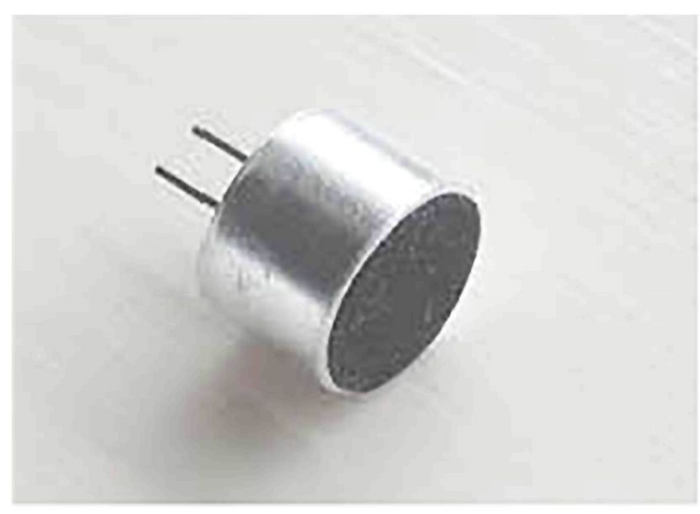

Fig. 1. Electret microphone capsule (sample).

rejection and are designed for general applications, particularly suitable for incorporation into portable equipment.

To perform the experiments, we purchased 100 microphone electret capsules, selecting 10 microphones for each temperature test.

In Table 1 the high sensibility and appropriate signal-noise ratio are highlighted, considering the small size and low price ( $2 \$$ ). Active low cost electronic devices are incorporated (FET amplifier) into the microphone capsules; therefore, the microphones need continuous voltage supply and a $5 \mathrm{~V}$ source was used for that function. Fig. 1 shows a single sample of an electret microphone capsule.

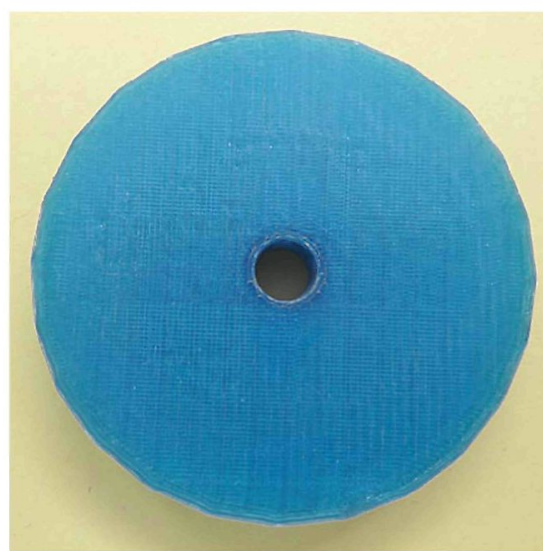

(a)

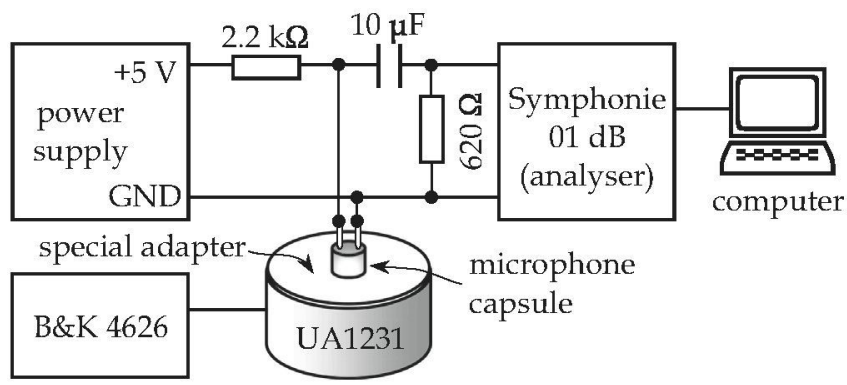

Fig. 3. Block diagram of the measurement setup.

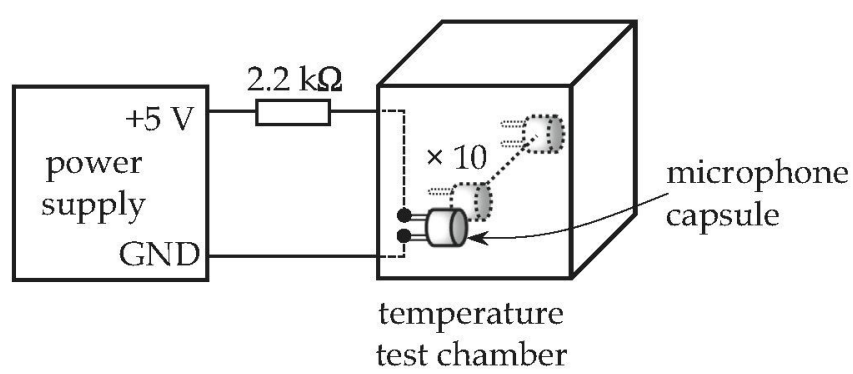

Fig. 4. Block diagram of the temperature tests.

\subsection{Measurement method}

\subsubsection{Sensitivity}

The parameter for analysis in our degradation experiments is microphone sensitivity, $S$

$S=\frac{\mathrm{V}}{\mathrm{p}}$,

where $V$ is the output voltage and $p$ is the acoustic pressure on the transducer. To generate the acoustic pressure $p$, the Bruel \& Kjaer 4226 Multifunction Acoustic Calibrator was used. This device emitted pure tones at the central frequencies of the octave bands from $31.5 \mathrm{~Hz}$ to $16 \mathrm{kHz}$ and additionally, at $12.5 \mathrm{kHz}$. For this reliability test, we applied to the microphones only the $94 \mathrm{~dB}$ and $114 \mathrm{~dB}$ Sound Pressure Levels (SPL). The former acoustic measurements were recorded during the degradation process, every time the 10 sample microphones were

Fig. 2. Microphone-calibrator special adapter (a) Top view (b) Bottom view.

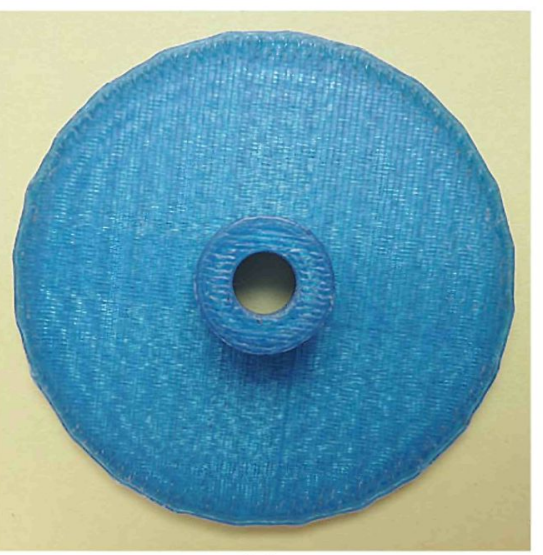

(b) 


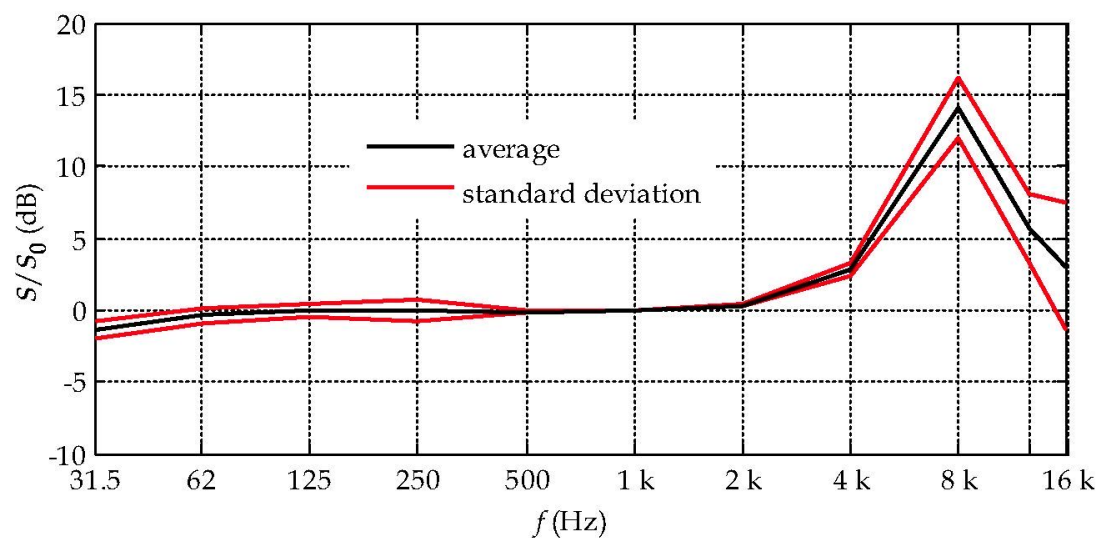

Fig. 5. Average frequency response of the total microphone population at $t=0$, prior to any temperature degradation.

removed from the temperature chamber (please refer to the next paragraph).

In the B\&K 4226 calibrator the clamping ring adapter model UA1231 was used to couple the $1 / 2^{\prime \prime}$ microphones to the acoustic emitter. To determine the variations in the sensitivity produced during the degradation process, it became necessary to guarantee uniform acoustic excitation to each microphone during the acoustic measurements; therefore, perfect mechanical coupling between the microphone and calibrator had to be achieved. As the microphone was $6 \mathrm{~mm}$ in diameter and different from the standard UA1231 hole, a specific adapter (plastic PLA, polylactide) had to be made using a 3D printer. Fig. 2 shows two views of this adapter.

Due to the shape and material of this adapter, a resonance of the set adapter-microphone was produced, which will be confirmed later. Although this resonance enhanced the pressure level around the $8 \mathrm{kHz}$ frequency, it had no effect on the degradation analysis.

Fig. 3 shows the sensitivity measurement block diagram.

\subsubsection{Temperature test}

First, the sensitivity of all the samples under test was recorded under laboratory climatic conditions, outside the temperature chamber. From now on this event is associated to $t=0$, where $t$ represents the test time. Next, the samples were placed in the chamber at the three different temperatures selected $(383 \mathrm{~K}, 398 \mathrm{~K}$ and $413 \mathrm{~K}$ ) for the different time intervals. Each temperature was applied simultaneously on the 10 different microphone capsules; therefore, there were $3 \times 10=30$ samples in total to test. The time span for each of the 10-microphone samples depended on the temperature, showing that the greater the time taken the lower was the temperature. The time intervals were cumulative in each microphone set.

The background acoustic level in the chamber was about $80 \mathrm{~dB} S P L$, covering all the audio spectrum.

Each electret microphone was biased with $5 \pm 0.2 \mathrm{~V}$ using a load resistor of $2.2 \mathrm{k} \Omega$ (Fig. 4). The power supply and resistors, one per microphone, were left outside the climatic chamber and only the electret microphones were placed inside the chamber. The chamber temperature accuracy was maintained at $\pm 1{ }^{\circ} \mathrm{C}$. A basic block diagram of the test configuration is shown in Fig. 4.

\section{Test results}

3.1. Variations in the frequency response of the microphones with temperature

The frequency response of the microphones selected was recorded according to the procedure described above, using the multi-frequency calibrator. In Fig. 5 the average frequency response of the total microphone population at $t=0$ was represented, along with the corresponding standard deviation (over 30 microphones). This graph shows that the magnitude that averaged is the relative sensitivity $S / S_{0}$, where $S$ is the sensitivity of each microphone and $S_{0}$ is its specific sensitivity at $1 \mathrm{kHz}$. This implies that in Fig. 5 only the frequency response variances with respect to $1 \mathrm{kHz}$ have been considered, so the three curves cross $0 \mathrm{~dB}$ at $1 \mathrm{kHz}$. On the other hand, the mean and standard deviation values of the 30-microphone sensitivity at $1 \mathrm{kHz}$ have been calculated with the following results:

$S_{0}($ mean $)=-42.5 \mathrm{~dB}($ re. $1 \mathrm{~V} / \mathrm{Pa})$ and

$S_{0}($ standard deviation $)=0.5 \mathrm{~dB}($ re. $1 \mathrm{~V} / \mathrm{Pa})$.

As evident from Fig. 5, the microphone population is highly homogeneous. The resonance at $8 \mathrm{kHz}$ is due to the acoustic conditions of the assembly with the multi-frequency calibrator, which has been confirmed with some frequency response measurement in an anechoic room of certain microphone units.

Fig. 6 shows the average degradation of the frequency response vs. time for all the microphones. The different figures represent the different conditions of temperature and excitation, SPL. Here, the degradation represented is the difference $S(f, t) / S(f, t=0)$ expressed in $\mathrm{dB}$. As evident, the degradation vs. frequency at each time point is basically uniform. Only high frequency around mechanical resonance exhibit deviations from this uniform degradation, especially in Fig. 6 (b). This may be attributed to a mechanical degradation with temperature of the microphone diaphragm or poor coupling to the calibrator in some tests.

\subsection{Degradation analysis at $1 \mathrm{kHz}$ and $94 \mathrm{~dB}$}

According to [4], the degradation analysis was conducted at $1 \mathrm{kHz}$ frequency and $94 \mathrm{~dB} S P L$ acoustic excitation.

The application of this degradation process stopped when the sensibility loss was $10 \mathrm{~dB}$ from the initial value or an accumulated test time of $29 \mathrm{~h}$.

The sensitivity for each microphone was adjusted to an exponential function (2) for all tests (Fig. 7) according to [5-6]:

$\frac{S(\mathrm{t})}{\boldsymbol{S}_{0}}=\mathrm{A} e^{-\frac{\mathrm{t}}{\tau(\mathrm{T})},}$

where $S(t)$ is the microphone sensitivity at time $t, S_{0}$ the sensitivity at time zero, $A$ is a dimensionless constant, $t$ the time in hours and $\tau(T)$ the temperature-dependent time constant. The initial point $(t=0)$ has not 
(a): $T=383 \mathrm{~K}, S P L=94 \mathrm{~dB}$

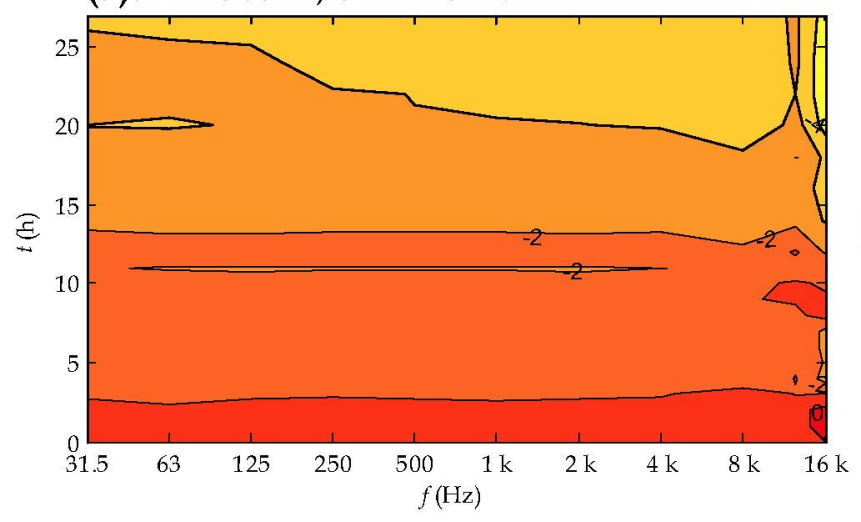

(c): $T=398 \mathrm{~K}, S P L=94 \mathrm{~dB}$

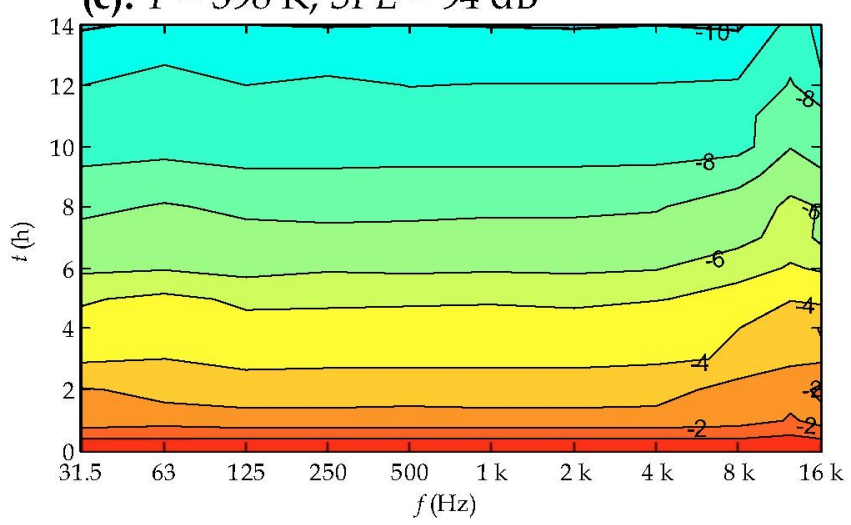

(e): $T=413 \mathrm{~K}, S P L=94 \mathrm{~dB}$

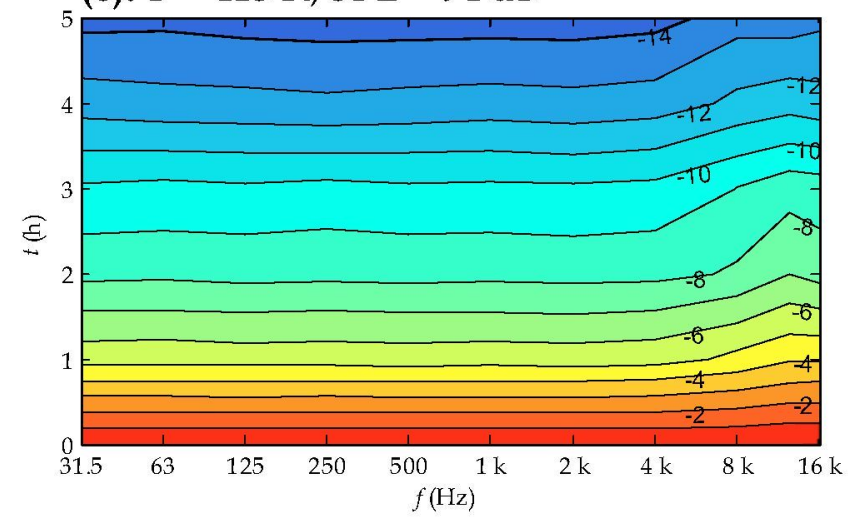

(b): $T=383 \mathrm{~K}, S P L=114 \mathrm{~dB}$

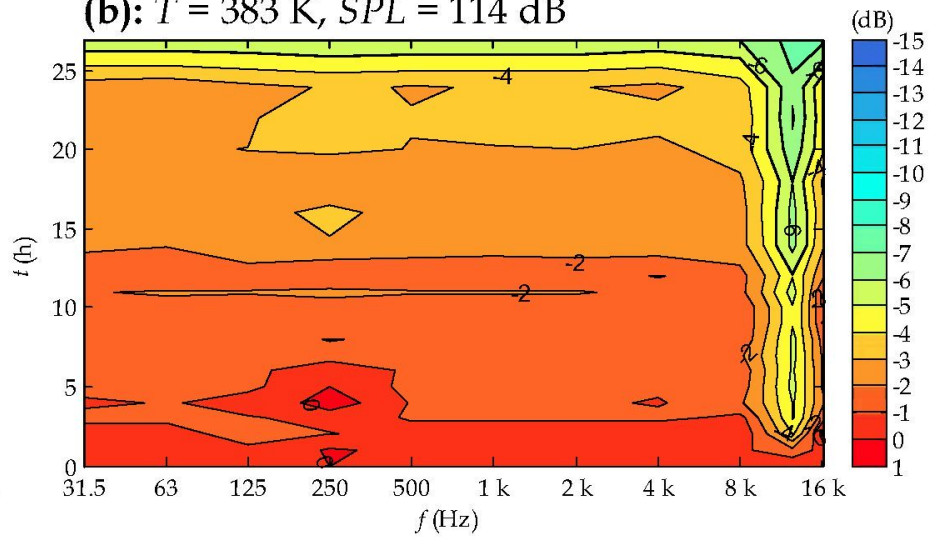

(d): $T=398 \mathrm{~K}, S P L=114 \mathrm{~dB}$
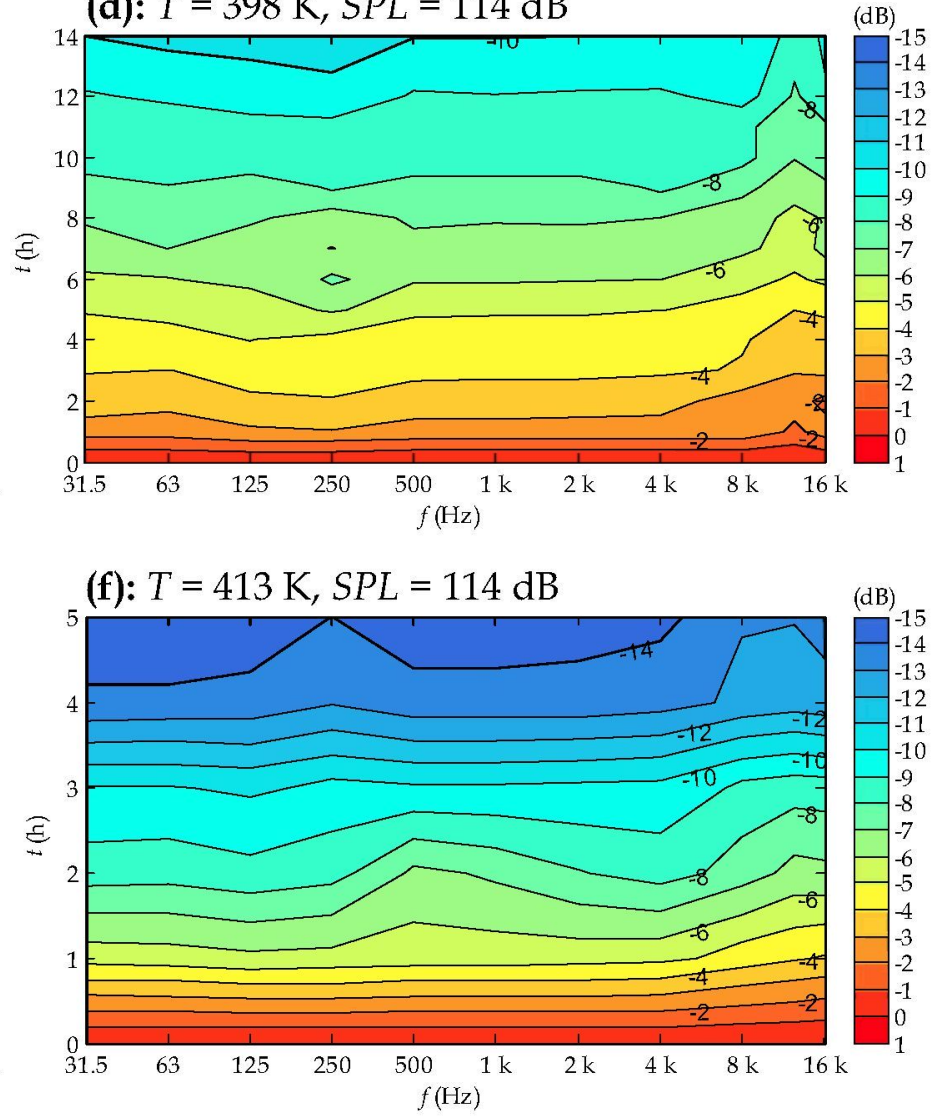

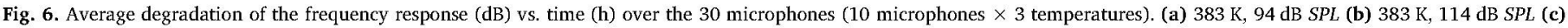
398 K, 94 dB SPL (d) 398 K, 114 dB SPL (e) 413 K, 94 dB SPL (e) $413 \mathrm{~K}, 114 \mathrm{~dB} S P L$.

been taken into account in the fit of the curves, since it has no degradation. This is the reason why all curves are below $S(t) / S_{0}=1$ at $t=0$.

Fig. 8 shows the curves of the average values for the three tests, at $383 \mathrm{~K}, 398 \mathrm{~K}$ and $413 \mathrm{~K}$.

Table 2 shows the values of $\tau(T)$ and $A$ according to (2) for each test as shown in Fig. 8. For the range of the temperature tested the variation of $A$ is low, and the time constant $\tau(T)$ decreases with increasing temperature, indicating that the higher the temperature the greater the degradation of the electret material.

Fig. 9 represents $\tau(T)$ vs $(k T)^{-1}$, corresponding to the Arrhenius model. The resulting $\tau$ function is shown in (3), which enables the time constant $\tau$ to be calculated at low ambient temperature.

$\tau(T)=3.27 \cdot 10^{-18} e^{\frac{1.47}{\mathrm{kT}}}$. 


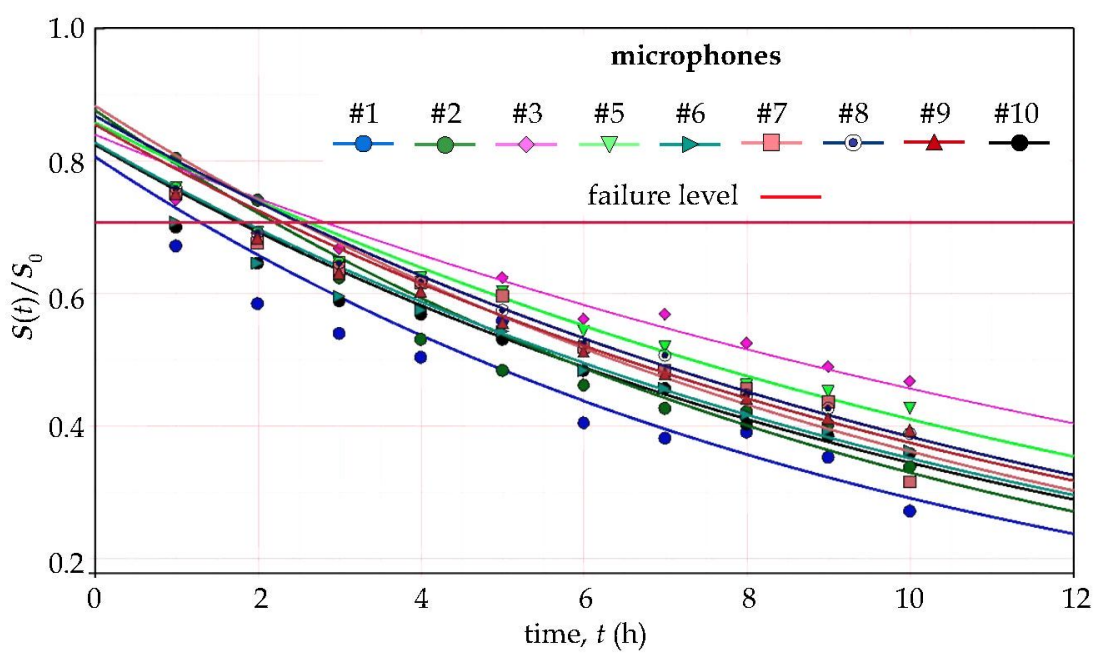

Fig. 7. For example, the exponential degradation for the $398 \mathrm{~K}$ test Each curve corresponds to one microphone. The horizontal line indicates failure $(-3 \mathrm{~dB})$. There will be two similar graphs for $383 \mathrm{~K}$ and $413 \mathrm{~K}$.

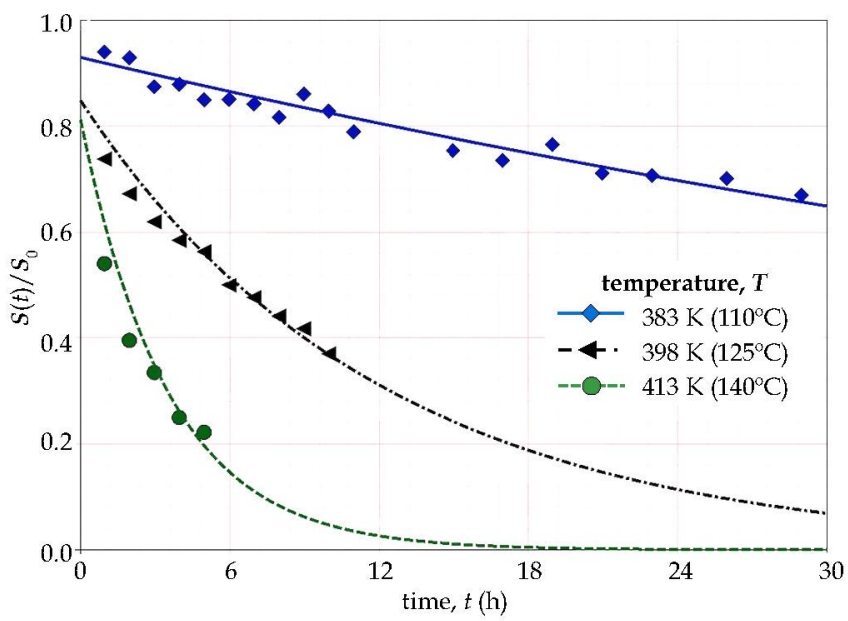

Fig. 8. Curves of the average values of sensitivity degradation at $383 \mathrm{~K}, 398 \mathrm{~K}$ and $413 \mathrm{~K}$.

Table 2

Values of $\tau(T)$ and $A$ according to (2) for each temperature.

\begin{tabular}{llll}
\hline$T(\mathrm{~K})$ & $A$ & $\tau(T)(\mathrm{h})$ & Accumulated time test $(\mathrm{h})$ \\
\hline 383 & 0.93 & 83.56 & 29 \\
398 & 0.85 & 11.94 & 11 \\
413 & 0.81 & 3.49 & 5 \\
\hline
\end{tabular}

\section{Reliability and life data analysis}

In this analysis, failure is defined as the sensitivity loss of $3 \mathrm{~dB}$ relative to the value at $t=0$. No catastrophic failure (which can be short or open circuit) has been detected in the tests. Degradation was detected only in the microphone sensitivity reduction.

Times to failure were determined in the $94 \mathrm{~dB} S P L$ tests because the $114 \mathrm{~dB}$ tests showed close similarity (see Fig. 6).

Fig. 7 shows sensitivity for each unit vs time in the $398 \mathrm{~K}$ test. The horizontal line marks the $3 \mathrm{~dB}$ sensitivity loss. The intersection of this line with each one of the exponential curves gives the time to failure for each microphone.

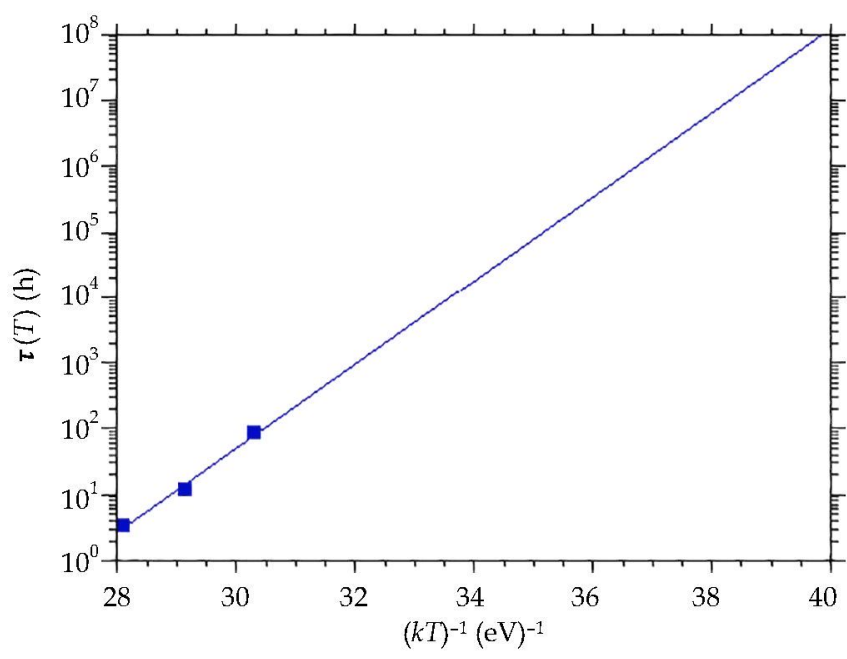

Fig. 9. Curve $\tau(T)$ vs $(k T)^{-1}$ as in (3) representing the Arrhenius model (temperature in kelvin).

Table 3 shows the time to failure for the three tests, in which the failure times decrease with temperature. The microphones \#4 (398 K) and \#8 (383 K) suffered a break in one of their terminals, due to poor handling, and as it was impossible to repair them they were removed from the test.

The life analysis is presented for the three different temperatures. Fig. 10 shows the Weibull plot for the degradation failures on the temperature test. The $y$-axis represents the unreliability function $F(t)$ and $x$-axis is the time to failure as shown in Table 4. These parameters were obtained using the maximum likelihood estimation method.

The 50th percentile obtained in Table 4 is presented in an Arrhenius plot in order to obtain a model for the temperature conditions presented here; this is shown in Eq. (4), [7] and Fig. 11, an regression coefficient $\mathrm{R}^{2}=0.98$ was obtained, showing a very good fit. The red line indicates the life at $300 \mathrm{~K}$ temperature.

$\mathrm{t}_{50 \%}=2.26 \cdot 10^{-22} \mathrm{e} \frac{1.74}{\mathrm{kT}}$,

where $t_{50 \%}$ is the 50th percentile, $k$ the Boltzmann constant $\left(8.62 \cdot 10^{-5} \mathrm{eV} \mathrm{K}^{-1}\right.$ ) and $T$ the absolute temperature (K); the activation 
Table 3

Time to failure for the different tests in hours.

\begin{tabular}{|c|c|c|c|c|c|c|c|c|c|c|}
\hline \multirow[t]{2}{*}{$T(\mathrm{~K})$} & \multicolumn{10}{|c|}{ \# microphone } \\
\hline & 1 & 2 & 3 & 4 & 5 & 6 & 7 & 8 & 9 & 10 \\
\hline 383 & 26.43 & 25.48 & 24.25 & 10.92 & 19.58 & 36.43 & 22.76 & - & 28.46 & 20.30 \\
\hline 398 & 1.28 & 2.19 & 2.63 & - & 2.40 & 1.55 & 2.48 & 2.33 & 2.22 & 1.44 \\
\hline 413 & 0.63 & 0.59 & 1.00 & 0.32 & 0.34 & 1.07 & 0.72 & 0.22 & 0.29 & 0.35 \\
\hline
\end{tabular}

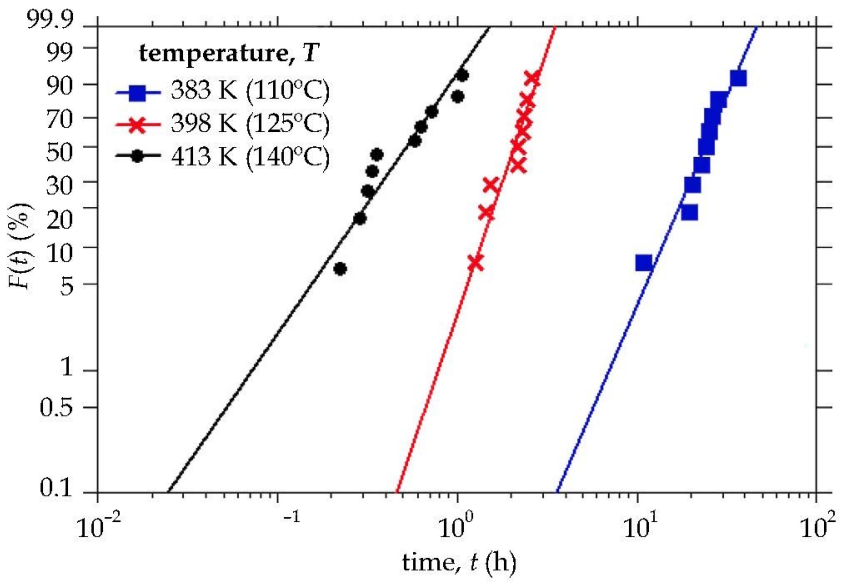

Fig. 10. Weibull plot for the temperature tests.

Table 4

Weibull parameters and 50th percentile for the temperature test

\begin{tabular}{llllll}
\hline $\mathrm{T}(\mathrm{K})$ & Sample size & $\begin{array}{l}\text { Number of } \\
\text { failures }\end{array}$ & $\begin{array}{l}\text { Weibull } \\
\text { shape } \\
\text { parameter }\end{array}$ & $\begin{array}{l}\text { Weibull scale } \\
\text { parameter }\end{array}$ & $\begin{array}{l}\text { Percentile } \\
50 \%(\mathrm{~h})\end{array}$ \\
\hline 383 & 9 & 9 & 3.45 & 26.51 & 23.84 \\
398 & 9 & 9 & 4.36 & 2.25 & 2.07 \\
413 & 10 & 10 & 2.15 & 0.62 & 0.52 \\
\hline
\end{tabular}

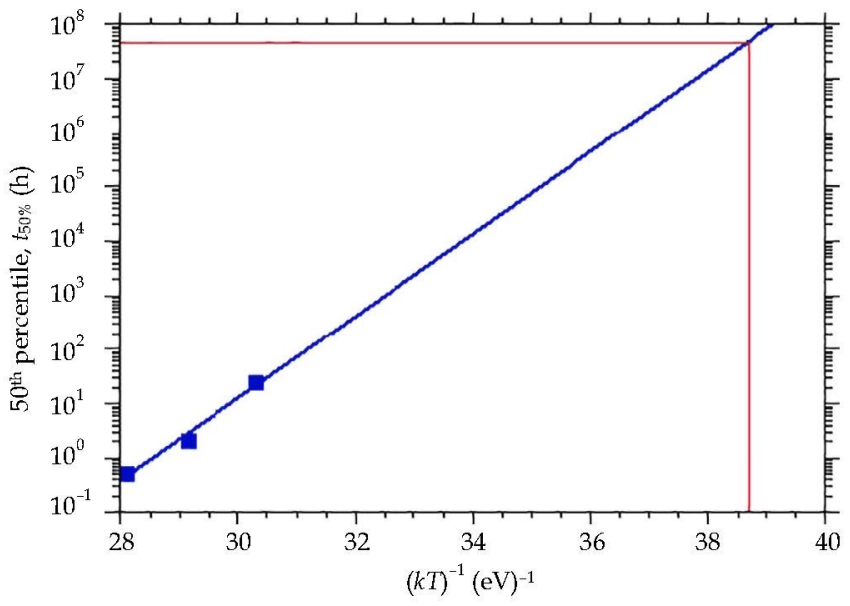

Fig. 11. Arrhenius plot. energy obtained is slightly higher than the one obtained by [8] $1.2 \mathrm{eV}$ and [9] $1.3 \mathrm{eV}$; this is likely because of the difference in the materials used in the electret. From Eq. (4), $t_{50} \%=4.49 \cdot 10^{7} \mathrm{~h}$. Upper Bound (confidence level $=90 \%$ ) $=2.95 \cdot 10^{6} \mathrm{~h}$ and Lower Bound (confidence level $=90 \%)=6.84 \cdot 10^{8} \mathrm{~h}$.

\section{Conclusions}

Degradation is independent of the frequency as shown in Fig. 6.

Exponential model is suitable for electret degradation (Fig. 7).

Different sound pressure levels were seen to produce similar degradation results; therefore, the influence of the excitation level is not significant (Fig. 6).

Arrhenius-Weibull model is suitable for the prediction of life times according to Section 4 (reliability and life data analysis), $\mathrm{R}^{2}=0.98$ was obtained.

The 50th percentile is $4.49 \cdot 10^{7} \mathrm{~h}$. The resulting life times are better than the findings reported in the earlier works available in the literature [7]. This is ascribed to the improved and modern technology, over time, of the electret materials. Activation energy obtained is higher $1.74 \mathrm{eV}$ than the one obtained by [8] $1.2 \mathrm{eV}$ and [9] $1.3 \mathrm{eV}$; this is likely because of the difference in the materials used in the electret.

\section{References}

[1] Y. Yasuno, Y.A. Riko, Chronological review of production and applications of electret condenser microphone for consumer use, 8th International Symposium on Electrets, (ISE 8), IEEE, 1994, pp. 943-948.

[2] G.M. Sessler, et al., Book metrics, Top. Appl. Phys. 33 (1987).

[3] Y. Yasuno, Y. Riko, Study of degradation by heat and heat-resistance property in electret condenser microphone, Electron. Commun. Jpn. 90 (5) (2007).

[4] IEC 61672-1 2002-05, Electroacoustics Sound Level Meters - Part 1: Specifications, (2018).

[5] L.A. Dissado, G. Mazzanti, G.C. Montanari, The role of trapped space charges in the electrical aging of insulating materials, IEEE Trans. Dielectr. Electr. Insul. 4 (5) (1997) 496-506.

[6] J. Van Turnhout, Thermally stimulated discharge of polymer electrets, Polym. J. 2 (2) (1971) 173-191.

[7] Kenzo Miura, Yoshinobu Yasuno, Long-term sensitivity changes in electret condenser microphones, J. Acoust. Soc. Japan (E) 18 (1) (1997) 29-35.

[8] Gerhard M. Sessler, James E. West, Foil-electret microphones, J. Acoust. Soc. Am. 40 (6) (1966) 1433-1440.

[9] Sten Hellström, A Reliability Study of Electronic Components and Electret Foils, Including Latent Failures Due to Submission to Electrostatic Discharges in a

Historical Retrospective, Mikroelektronik och informationsteknik, 2003 ( $\mathrm{PhD}$ diss.). 